\title{
Avalanche danger forecast in little study territories using GIS
}

\author{
Natalia V. Kondratyeva ${ }^{1}$, Islam Z. Shidugov ${ }^{1}$ \\ ${ }^{1}$ Alpine Geophysical Institute, Nalchik, Russia
}

\begin{abstract}
Methods for determining the zone of avalanche initiation using tools are proposed ArcGis 10.4.1. The process of creating a raster of the slope of heights and later on the territory itself for dividing them into existing gradations is described. Demonstrated the process of determining the area of avalanche foci and determining the boundaries of the avalanche collection. The areas of avalanche origination within the avalanche collection have been corrected. The method of creating a map of the exposure of slopes in order to identify the area of accumulation and drift of snow, its dynamics and characteristics of snow melting is shown. Based on this, it becomes possible to determine the maximum height of avalanche initiation and the range of the avalanche ejection.
\end{abstract}

Keywords

Avalanche collection, range of avalanche emission, avalanche height, GIS, raster.

\section{Introduction}

Snow avalanches are included in the list of dangerous natural phenomena that have a negative impact on buildings and structures. Therefore, when designing various objects in the mountainous territories of Russia, it is necessary, first of all, to assess the danger of the impact of an avalanche on the object in order to prevent damage from an avalanche manifestation.

To date, according to the requirements of regulatory documents, an avalanche hazard assessment in the area under consideration must be carried out according to SP 428.1325800 .2018 "Engineering surveys for construction in avalanche-prone areas. General requirements" [1]. According to this SP, it is necessary to assess the characteristics of the morphometry of the zones of initiation, transit, deposition of avalanches, elevation marks, maximum and average angles of inclination of avalanche collection, slope exposure and the range of its variation. These issues are not fully worked out in the modern technical literature [2].

\section{Proposed solution}

This paper shows an example of the use of GIS to find the required morphometric parameters in the analysis of avalanche hazard at the site of the design of the facility.

Intensive development of computer and information technologies has made it possible to create a variety of software that allows you to solve various physical and geographical problems

SDM-2021: All-Russian conference, August 24-27, 2021, Novosibirsk, Russia

$\bigoplus$ vgikbr@yandex.ru (N.V. Kondratyeva)

(c) (1) $\odot 2021$ Copyright for this paper by its authors. Use permitted under Creative Commons License Attribution 4.0 International (CC BY 4.0).

CEUR Workshop Proceedings (CEUR-WS.org) 
in accordance with modern requirements. In this regard, there is a need for a detailed mastering of the tools developed by GIS $[3,4,5]$ to determine the morphometric and physical-geographical characteristics of territories. In this work, this is the ArcGis GIS program, version 10.4.1.

In general, the role of GIS technologies in avalanche studies is reduced to the synthesis of knowledge about the relief, in order to determine the possibility of avalanches. To do this, in the GIS environment, we propose to solve the following tasks.

1) Digitize ready-made topographic paper maps and satellite images, and create new map projects taking into account new designed objects.

2) Determine the zones of avalanche initiation (avalanche foci).

When solving this problem, it is necessary to have a vector large-scale (at least M 1:5000) topographic base (horizontals, if there are watercourses, then a vector layer of rivers) of the mountainous area under consideration. Then, using the tool group: Spatial Analyst $\rightarrow$ Interpolation $\rightarrow$ TopoToRaster, a raster (raster is an image synthesized by a computer and built on the screen) of heights is created. Then, using the resulting raster of heights and tools: Spatial Analyst $\rightarrow$ Surface $\rightarrow$ Slope, create a raster of slopes of the territory (the arrow indicates further).

According to [1], avalanches are formed at slope angles from 25 to $60^{\circ}$; from 0 to $20^{\circ}-$ territories where avalanche initiation is impossible, and if they are located under slopes where avalanches can form, these are territories where avalanche deposition zones are located; $20-25^{\circ}$ - slopes where the likelihood of avalanche formation is extremely low; more than $60^{\circ}-$ slopes on which snow does not hold. Thus, further in the properties of the raster slopes in the tab "Symbols" $\rightarrow$ "Classification" it is necessary to divide the slope angles into the above gradations.

On the raster of slopes, there are areas with the most favorable conditions for the occurrence of avalanches $\left(25-60^{\circ}\right)-$ avalanche foci. The tools: Spatial Statistics $\rightarrow$ Utilities $\rightarrow$ Calculate Area determine the area of avalanche foci, i.e. areas where snow accumulation occurs.

3) The next step is to determine the boundaries of the avalanche collection. To do this, using the tool - create an object (Create Features), in the editing mode, using a vector topographic base and a raster of heights, an avalanche collection is outlined: the upper boundary is determined along the line of the summit ridge, the lateral boundaries go along the ridges separating negative landforms , the lower boundary of the avalanche collection (the foot of the slope) is determined, taking into account the constructed raster of slopes, as a site under the slope, where the angles are from 0 to $20^{\circ}$.

After creating the boundaries of avalanches, the areas where avalanches originate within the boundaries of avalanches are corrected.

Figures 1, 2 show examples of the constructed rasters of heights (the legend indicates the gradations of the heights) and average slopes (the legend indicates the gradations of the average slopes) with the boundaries of avalanche collections and avalanche foci, as well as in the figures, as the boundary of the projected object.

4) In order to study the spatial distribution of snow cover - to identify areas of accumulation and drift of snow, its dynamics, characteristics of snow melting, slope exposure maps are created. To do this, it is proposed to use the constructed raster of heights and a group of tools: Spatial Analyst $\rightarrow$ Surface $\rightarrow$ Exposure, the legend shows the compass directions of exposure (Figures 3, 4). 


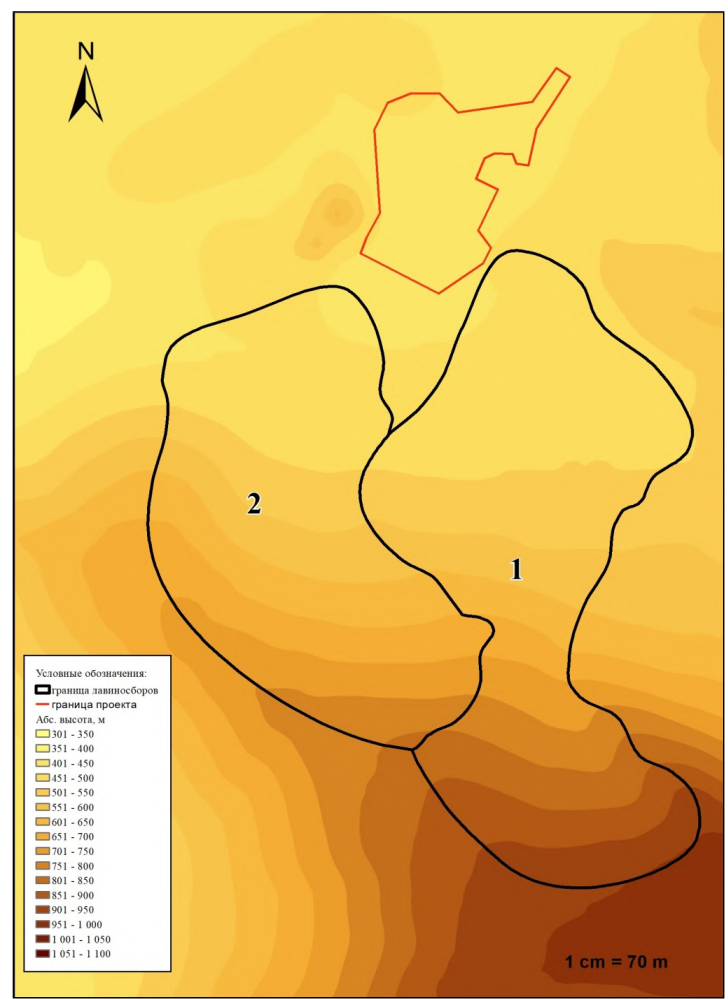

Figure 1: Raster of heights with the boundaries of avalanches.

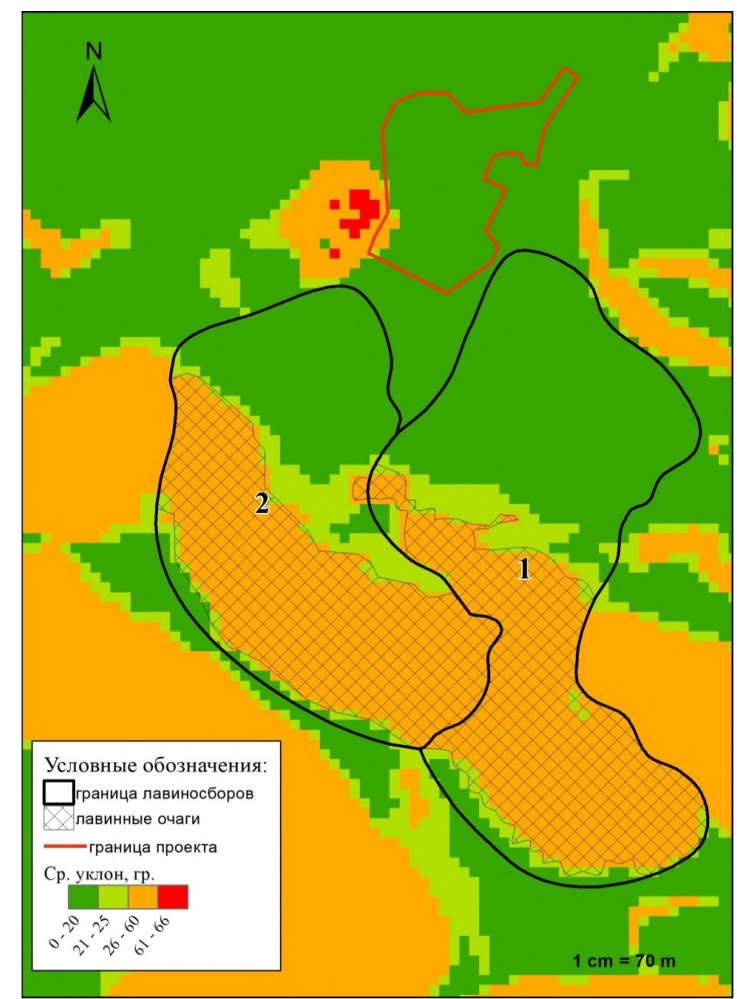

Figure 2: Raster of average slopes with marked boundaries of avalanche gatherings and avalanche centers in them.

5) It is also possible in the GIS environment to determine the range of an avalanche ejection, for this a longitudinal profile of the avalanche collection is built - the average line of the avalanche path from the place of avalanche separation to the foot of the slope. To do this, use a tool - a profile graph (Profile View).

6) Using the tools: 3D Analyst $\rightarrow 3 \mathrm{D}$ objects $\rightarrow$ Add $\mathrm{Z}$ information, you can determine the maximum height of the avalanche origin, the avalanche itself, the lowest $Z$-value, the average $Z$-value, the minimum slope value, the maximum slope value, the average slope value and etc.

7) For a more visual perception of the above actions, it is possible to view the boundaries of avalanches on a detailed satellite image. To do this, you need to get a satellite image of the area in question in any format supported by GIS ArcGis 10.4.1., For example PNG, JPG, TIF, etc. The satellite image must be accompanied by a file storing information about the spatial reference. Further, this data is added to the active data frame of the map, for this, the following tools are used: Add data $\rightarrow$ Connect to folder, after which the appropriate directory is selected. Then by double-clicking the map file is added to the active frame, after which it becomes possible to use this space-mock as a raster.

Thus, the goals of creating GIS-projects, including avalanche topics, are reduced to determining the state of the investigated area for the occurrence of an avalanche hazard. These are: providing planning, design, controlling organizations with information about the spread 


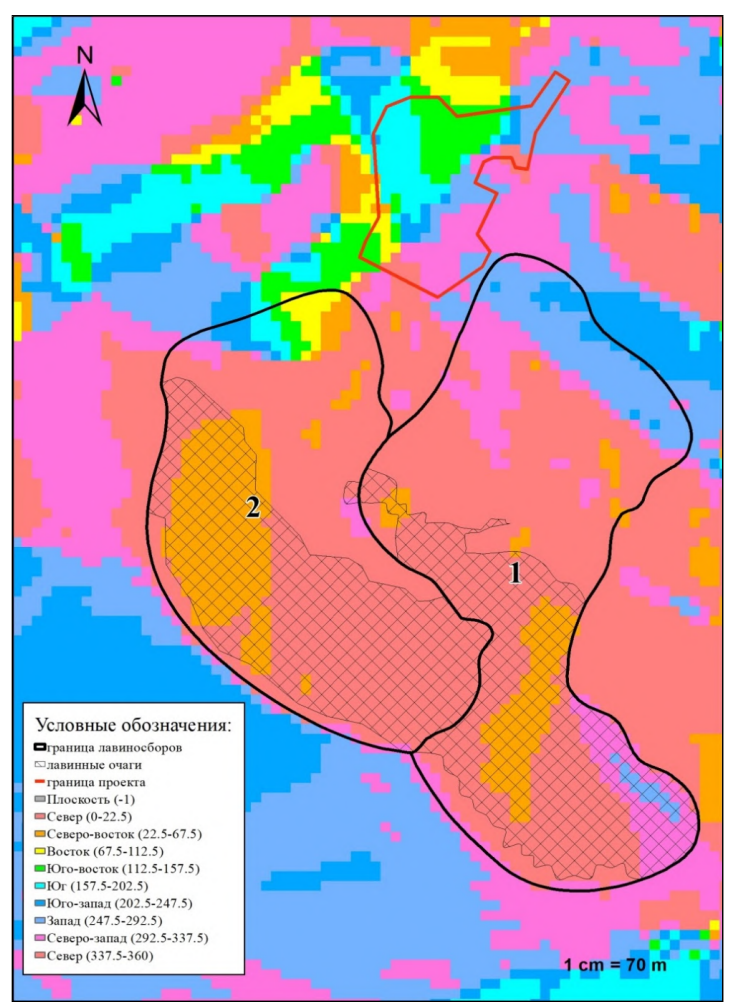

Figure 3: Raster of exposures of slopes with avalanche boundaries.

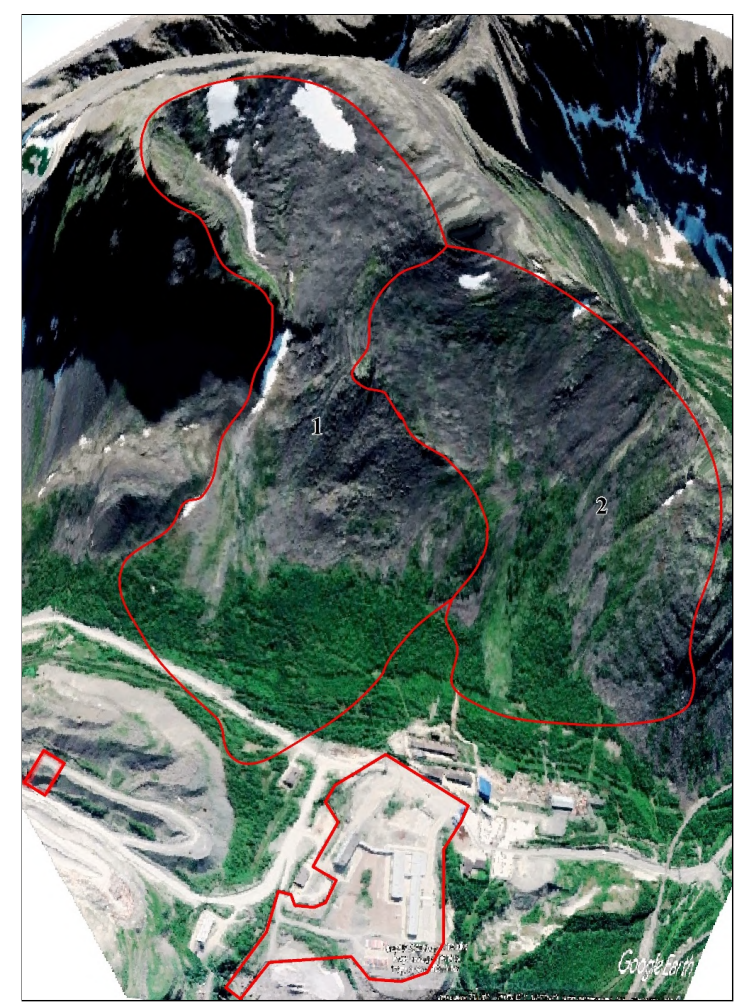

Figure 4: Satellite image of slopes with avalanche boundaries.

of natural hazards, creating a land cadastre, choosing the best places for the construction of linear and areal facilities. Further, using the data obtained using GIS, it is necessary to perform avalanche calculations provided for by SP 428.1325800 .2018 "Engineering surveys for construction in avalanche-prone areas. General requirements". In particular: dynamic parameters of dry and wet avalanches, avalanche volumes, etc.

\section{Conclusion}

The proposed use of GIS (ArcGis program, version 10.4.1.) Allows for an analysis of the avalanche hazard assessment for unexplored territories. In this case, it seems possible:

- to determine the morphometric parameters of avalanches;

- range of avalanches;

- to assess the impact of avalanches and air waves on existing and projected facilities.

If data from field surveys and long-term observations are available, the results obtained must be clarified and, if necessary, corrected. 


\section{References}

[1] SN 517-80: Instruction for the design and construction of avalanche protection structures. Moscow: Stroyizdat, $1980.15 \mathrm{p}$

[2] VSN 02-73 Instructions for the calculation of avalanche loads in the design of structures. Moscow: Gidrometeoizdat, 1973. 22 p.

[3] Brown L.A. History of geographical maps. Moscow: Tsentrpoligraf, 2006 .- 479 p.

[4] Zhurkin I.G., Shaytura S.V. Geoinformation systems. Moscow: Kudits-press, 2009. 272 p.

[5] DeMers M.N. Geographic information systems. Fundamentals: Translate from English. Moscow: Date+, 1999. 489 p. 